\title{
Lack of Influence of TP53 Arg72Pro and 16bp Duplication Polymorphisms on Risk of Breast Cancer in Iran
}

\author{
Sahar Gohari-Lasaki ${ }^{1}$, Jalal Gharesouran ${ }^{1}$, Morteza Ghojazadeh ${ }^{2}$, Vahid \\ Montazeri $^{3}$, Seiied Moitaba Mohaddes Ardebili ${ }^{1 *}$
}

\begin{abstract}
TP53 is assumed to be a very important tumour suppressor gene, as illustrated by recent reports that have shown effects of its polymorphisms on breast cancer risk. Arg72Pro and PIN3(16bp duplication) polymorphisms are proposed to have an effective role in structural changes of p53 and have therefore attracted interest as a risk factor for breast cancer in different populations. The aim of this study was to examine and determine whether p53 codon 72 and PIN3 Ins16 bp may be associated with an increased risk for breast cancer in female patients from the northwest of Iran. Genotyping was performed by PCR-RFLP (polymerase chain reaction-restriction fragment length polymorphism) method for a total of 100 women with breast cancer and 100 healthy women without any background of cancer, focusing on the TP53 Arg72Pro-16Del/Ins haplotypes and the combined genotypes. The results in this study established no statistical significant distinctions between the genotypes and allele frequency were found for Arg72Pro and PIN3 Ins 16 bp polymorphisms between patients and controls.
\end{abstract}

Keywords: Breast cancer - polymorphism - TP53 - codon 72 - PIN3 Ins16bp - Iran

Asian Pac J Cancer Prev, 16 (7), 2971-2974

\section{Introduction}

TP53 is a tumour suppressor gene with 11 exons on chromosome 17p13.TP53 gene encodes $\mathrm{p} 53$ protein, which is comprised of 393 amino acids and acts as transcription factor, having an essential role in genome integrity and a well established role in preventing tumorigenesis (Zhou et al., 2012). Somatic mutations of TP53 that result in malfunction or absence of $\mathrm{p} 53$ pathway arise from lesions during carcinogenesis. The loss of correct function of p53 is associated with many types of cancer: Sporadic cancers, correlated with mutations in somatic cells (Vijayaraman et al., 2012).

The mutations terminated in tumorigenesis are single base substitutions that result in amino acid substitutions in the DNA-binding domain of p53 (Whibley et al., 2009). The most repetitive genetic occurrences between the tumour suppressors is in p53 that effect the cell cycle regulation in the G1/S checkpoint for DNA repair (Sharma et al., 2014).

P53 induces many cellular stress pathways, such as hypoxia, DNA damage, telomere shortening and oncogene activation (Zhou et al., 2012). Mutations in the p53 gene impairing its function are detected in breast cancer and associated with clinical phenotypes (Alawadi et al., 2011). Polymorphisms in this gene occur in both coding and noncoding sequences, however, $90 \%$ of the polymorphisms occurred in the noncoding sequences (Whibley et al., 2009). Typically, TP53 exons 5-9 consists of the zincfinger domain and transactivating domain that are mutational hotspots. Missense substitutions in the TP53 mutation are non random along the molecules, particularly the central DNA-binding-domain and therefore the affect of TP53 transcriptional activity to various degrees.

Wild type p53 protein has a short life span but the mutant forms have a longer half-life and exhibit wild type behaviour, resulting in the accumulation and overexpression of mutant p53 protein, illustrated by immunohistochemistry assays of abnormalities in the neoplasm (Bellini et al., 2012). One of the polymorphic variations of TP53 that may directly raise the individuals susceptibility to breast cancer is on codon 72 in exon 4 in a proline-rich region that is necessary at the protein level function for apoptosis (Hu et al., 2010). Polymorphism in codon 72 , which is carried by $20 \pm 40 \%$ of the population, changes an arginine to proline substitution (Sjalander et al., 1996; Samson et al., 2007). Polymorphisms in codon 72 (CCC or CGC) TP53 gene were found the in different cancers like colorectal cancer, lung cancer, bladder cancer and Breast cancer (Hu et al., 2010). In addition to the numerous coding region mutations, the p53 gene also contains various polymorphisms and intronic mutations Polymorphisms, such as a 16bp insertion (Ins) in intron 3 localized at nucleotide 11951 (PIN3 Ins 16bp (Hrstka

${ }^{1}$ Department of Medical Genetics, ${ }^{2}$ Department of RDCC, ${ }^{3}$ Department of General Surgery, School of Medicine, Hematology and Oncology Research Center, Tabriz University of Medical Sciences, Tabriz, Iran*For correspondence: mohaddesmo@yahoo.com 
et al., 2009). In the non-coding region of TP53, affect expression levels of p53 and its function (Guleria., 2012).

The role of 16bp polymorphisms in breast cancer is controversial. The involvement of the intronic PIN3 Ins $16 \mathrm{bp}$ variant in breast carcinogenesis is not well defined and there is therefore little data for specific human population available. Some studies have reported that PIN3 Ins 16bp polymorphism is associated with an increased risk for breast cancer (Alawadi et al., 2011).

\section{Materials and Methods}

The mean age of the selected participants was 45 years, patients of the Nejat Hospital, Tabriz, Iran. Peripheral blood samples were collected from 100 women diagnosed with sporadic breast cancer.Additionally, blood samples were also collected from 100 healthy cancer-free females. The samples were collected in vacutianer tubes containing EDTA anticoagulant and the samples were stored at $4^{\circ} \mathrm{C}$ until analysis. The blood samples were drawn and collected upon completion of the written informed consents and signed by the patient. A DNA sample was extracted from $2 \mathrm{cc}$ of blood, the salting out method was utilized for this purpose.

\section{PCR amplification of exon 4 (codon72)}

The size of the PCR amplified with a forward primer 5'-CTGGTAAGGACAAGGGTTGG-3' and reverse primer 5'-ACTGACCGTGCAAGTCACAG-3' in 25 $\mu$ lreaction volume containing, $1.25 \mu \mathrm{l}$ of each primer, 0.5 $\mu \mathrm{l}$ of dNTPs, $1 \mu \mathrm{l}$ of $\mathrm{MgCl} 2,2.5 \mu \mathrm{l}$ of a standard PCR buffer, $0.4 \mu$ l of Taq polymerase and $3 \mu$ of DNA template. Science Protocol was Touchdown PCR Thermal cycling conditions were the following: $95^{\circ} \mathrm{C}$ for $2 \mathrm{~min}, 95^{\circ} \mathrm{C}$ for $30 \mathrm{~s}, 63.3^{\circ} \mathrm{C}$ decrease $0.5^{\circ} \mathrm{C}$ per cycle, $30 \mathrm{sec}, 72^{\circ} \mathrm{C}$ for $40 \mathrm{~s}$, followed by 14 step cycles of extension, $95^{\circ} \mathrm{C}$ for $30 \mathrm{~s}, 56^{\circ} \mathrm{C}$ for $30 \mathrm{~s}, 72^{\circ} \mathrm{C}$ for $40 \mathrm{~s}$, repeated in 19 cycles at $72^{\circ} \mathrm{C}$ for $5 \mathrm{~min}$. RFLP (Restriction fragment length polymorphism) was performed with $0.1 \mu \mathrm{l}$ of BstU1 at $37^{\circ} \mathrm{C}$ for $24 \mathrm{~h}$. The digestion products were then resolved on a $8 \%$ polyacrylamid gel. A single 396 bp bond for mutant homozygote's (C/C), 165 bp, 231 bp, 396 bp bonds was produced from heterozygotes $(\mathrm{G} / \mathrm{C})$ and $165 \mathrm{bp}, 231$ bp bonds from homozygote's (G/G).

\section{PCR Amplification of Intron3 16 bp duplication}

The intron 3 16bp duplication was amplified with forward primer 5'-TGGGACTGACTTTCTGCTCTT-3' and reverse primer 5' -TCAAATCATCCATTGCTTGG-3' .PCR products was $180 \mathrm{bp}$ or $196 \mathrm{bp}$. PCR products were resolved on a $8 \%$ polyacrylamide gel and then both bonds were detected in the stained with $\mathrm{AgNO} 31 \%$ and $\mathrm{NaOH}$ $37.5 \%$. DNA from wild-type allele showed a $180 \mathrm{bp}$ (no duplication) and allele with duplication demonstrated at 196 bp(16- bp duplication).

\section{Results}

Polymorphisms R72P in TP53 gene were analyzed for a total of 100 sporadic breast cancer patients and 100 women cancer-free. The age ranged from 20 to 70 years of age, in group- cases and control-groups. According to

Table 1. Allele and Genotype Frequencies of P53 Polymorphisms in Breast Cancer Patients and Healthy Women

\begin{tabular}{|c|c|c|c|c|c|}
\hline Polymorphisms & Cases n (\%) & Controls n (\%) & OR(95\%CI) & P-Value & Codon 72 Arg/Pro \\
\hline \multicolumn{6}{|l|}{$\overline{\text { Genotypes }}$} \\
\hline \multicolumn{6}{|l|}{ Codon 72 Arg/Pro } \\
\hline Arg/Arg & $31(31)$ & $31(31)$ & Ref & Ref & \\
\hline \multicolumn{6}{|l|}{ Genotypes } \\
\hline Arg/Pro & $48(48)$ & $57(57)$ & 1.18 & $(0.63-2.22)$ & 0.63 \\
\hline Arg/Arg & $31(31)$ & $31(31)$ & Ref & Ref & \\
\hline Pro/Pro & $21(21)$ & $12(12)$ & 0.5 & $7(0.24-1.35)$ & 0.27 \\
\hline Arg/Pro & $48(48)$ & $57(57)$ & 1.18 & $(0.63-2.22)$ & 0.63 \\
\hline \multicolumn{6}{|l|}{ Alleles } \\
\hline Pro/Pro & $21(21)$ & $12(12)$ & 0.57 & $(0.24-1.35)$ & 0.27 \\
\hline Arg & $110(55)$ & $119(59.5)$ & Ref & Ref & \\
\hline Alleles Pro & $90(45)$ & $81(40.5)$ & 0.83 & $(0.56-1.23)$ & 0.36 \\
\hline Arg & $110(55)$ & $119(59.5)$ & Ref & Ref & \\
\hline Pro & $90(45)$ & $81(40.5)$ & 0.83 & (0.56-1.23) & 0.36 \\
\hline \multicolumn{6}{|l|}{ Intron3 Ins 16 bp } \\
\hline \multicolumn{6}{|l|}{ Genotypes } \\
\hline \multicolumn{6}{|l|}{ Intron3 Ins 16 bp } \\
\hline A1A1 & $53(53)$ & $60(60)$ & Ref & Ref & \\
\hline \multicolumn{6}{|l|}{ Genotypes } \\
\hline $\mathrm{A} 1 \mathrm{~A} 2$ & $38(38)$ & $37(37)$ & 0.68 & $(0.47-1.54)$ & 0.65 \\
\hline $\mathrm{A} 1 \mathrm{~A} 1$ & $53(53)$ & $60(60)$ & Ref & Ref & \\
\hline $\mathrm{A} 2 \mathrm{~A} 2$ & $9(9)$ & $3(3)$ & 0.29 & $(0.07-1.14)$ & 0.07 \\
\hline $\mathrm{A} 1 \mathrm{~A} 2$ & $38(38)$ & $37(37)$ & 0.68 & $(0.47-1.54)$ & 0.65 \\
\hline \multicolumn{6}{|l|}{ Alleles } \\
\hline $\mathrm{A} 2 \mathrm{~A} 2$ & $9(9)$ & $3(3)$ & 0.29 & $(0.07-1.14)$ & 0.07 \\
\hline $\mathrm{A} 1$ & $144(72)$ & $157(78.5)$ & Ref & Ref & \\
\hline Alleles A2 & $56(28)$ & $43(21.5)$ & 0.74 & (0.44-1.11) & 0.13 \\
\hline A1 & $144(72)$ & $157(78.5)$ & Ref & Ref & \\
\hline $\mathrm{A} 2$ & $56(28)$ & $43(21.5)$ & 0.74 & $(0.44-1.11)$ & 0.13 \\
\hline
\end{tabular}


TP53 Arg72Pro and 16bp Duplication Polymorphisms and Risk of Breast Cancer

Table 2. Genotype Frequencies between R72P, PIN3 Ins16bp Polymorphisms

\begin{tabular}{|c|c|c|c|c|}
\hline \multirow{3}{*}{$\begin{array}{l}\text { Genotypes } \\
\text { RR A1A1 } \\
\text { Patients }\end{array}$} & \multicolumn{2}{|c|}{ Frequency (\%) } & \multicolumn{2}{|c|}{ Breast cancer risk } \\
\hline & $24(24 \%)$ & $22(22 \%)$ & Ref & Ref \\
\hline & Controls & $\mathrm{OR}(95 \% \mathrm{CI})$ & $\mathrm{P}$-value & \\
\hline RR A1A2 & $5(5 \%)$ & $6(6 \%)$ & $1.3(0.35-4.9)$ & 0.68 \\
\hline RR A2A2 & $0(0 \%)$ & $2(2 \%)$ & - & \\
\hline RR A1A1 & $24(24 \%)$ & $22(22 \%)$ & Ref & Ref \\
\hline RP A1A1 & $22(22 \%)$ & $35(35 \%)$ & $1.73(0.79-3.81)$ & 0.16 \\
\hline RR A1A2 & $5(5 \%)$ & $6(6 \%)$ & $1.3(0.35-4.9) 0.68$ & \\
\hline RP A1A2 & $21(21 \%)$ & $22(22 \%)$ & $1.14(0.49-2.62)$ & 0.75 \\
\hline RR A2A2 & $0(0 \%)$ & $2(2 \%)$ & - & - \\
\hline RP A 2 A2 & $4(4 \%)$ & $1(1 \%)$ & $0.27(0.02-2.63)$ & 0.23 \\
\hline RP A1A1 & $22(22 \%)$ & $35(35 \%)$ & $1.73(0.79-3.81)$ & 0.16 \\
\hline PP A1A1 & $6(6 \%)$ & $2(2 \%)$ & $0.36(0.06-1.99)$ & 0.23 \\
\hline RP A1A2 & $21(21 \%)$ & $22(22 \%)$ & $1.14(0.49-2.62)$ & 0.75 \\
\hline PP A $1 A 2$ & $10(10 \%)$ & $6(6 \%)$ & $0.65(0.2-2.1)$ & 0.47 \\
\hline RP A $2 A 2$ & $4(4 \%)$ & $1(1 \%)$ & $0.27(0.02-2.63)$ & 0.23 \\
\hline PP A2A2 & $4(4 \%)$ & $0(0 \%)$ & $0.85(0.73-0.99)$ & 0.065 \\
\hline PP A1A1 & $6(6 \%)$ & $2(2 \%)$ & $0.36(0.06-1.99)$ & 0.23 \\
\hline PP A $1 A 2$ & $10(10 \%)$ & $6(6 \%)$ & $0.65(0.2-2.1)$ & 0.47 \\
\hline PP A2A2 & $4(4 \%)$ & $0(0 \%)$ & $0.85(0.73-0.99)$ & 0.065 \\
\hline
\end{tabular}

the SPSS16 test no significant differences between the allele frequencies were noted for R72P polymorphism in the sporadic breast cancer patients and the control-group analyzed in this study. The proportion of RR, PP and RP genotypes in these patients were found to be $31 \%, 21 \%$ and $48 \%$, respectively, as compared to $31 \%, 12 \%$ and $57 \%$ in the control-group. The frequencies of mutant allele in the sporadic breast cancer patients was $45 \%$ and in the control-group $40 \%$, no statistically significant associations were noted in the presence of the p53 Pro allele and the increased risk of breast cancer $(\mathrm{p}<0.19)$ (Table 1). The frequencies of TP53 PIN3 Ins 16bp variant genotypes A1A1(180 bp in individuals homozygous for wild- type allele), A1A2 (180 bp and $196 \mathrm{bp}$ in heterozygotes) and A2A2 (196 bp individuals for homozygous mutant) in the sporadic breast cancer patients were found to be $53 \%$, $38 \%$ and $9 \%$ respectively, as compared to $60 \%, 37 \%$ and $3 \%$ in the control-groups. No significant differences were observed in the genotypes and alleles frequency in the breast cancer patients versus the healthy controlgroups $(\mathrm{p}<0.17)$. The frequencies of A2A2 genotype were common in the breast cancer patients than in the controlgroups (9\% vs. 3\%). The statistical analysis of the two polymorphisms demonstrated no differences of genotypes and alleles frequency in the breast cancer patients versus the healthy women (Table 2).

\section{Discussion}

A number of studies revealed high risk of breast cancer development due to $16 \mathrm{bp}$ duplication in intron3, while others studies failed to demonstrate any association between the duplication and cancer, and the differentiation in the results are in all probability, due to the ethical factors (Berrada et al., 2013). A number of studies indicated that women with these polymorphisms have an increased risk for developing breast cancer (Costa et al., 2007; Bellini et al., 2012). However, others studies, found no association between the R72P and intron 3 (+16 bp) duplication and the risk of breast cancer (Siddique et al., 2005; Hrstka et al., 2009; Alawadi et al., 2011). The investigative purpose and primary findings in this study was to evaluate R72P of TP53 and PIN3 Ins 16 bp polymorphisms in women with sporadic breast cancer versus the healthy women and the possible link between these polymorphisms and risk of developing breast cancer in this population (Alawadi et al., 2011).The TP53 Arg allele was preferentially expressed in most heterozygote breast cancer patients, suggesting that TP53 Arg expression correlates with breast cancer development.Various studies have demonstrated that healthy Asian heterozygous individuals (Tp53 Arg/Pro) tend to preferentially express the Tp53 Arg allele at the RNA level (Siddique et al., 2005). Reports from earlier studies suggest that the Tp53 Arg were associated with cancer predisposition (Sjalander et al., 1996; Ainsley Weston and James H. Godbold 1997; Papadakis et al., 2000; Bergamaschi et al., 2003) and further implied that even if the Tp53 Arg form might be capable of inducing and enhancing the formation of apoptosis, that this may not be sufficient in preventing the formation of cancer. This study selected a total of 100 patients for participation in this research, the frequency of $\mathrm{R}$ allele in the breast cancer patients were $55 \%$ and $\mathrm{P}$ allele was $45 \%$ and $59.5 \%$ of $\mathrm{R}$ allele and $40.5 \%$ for P allele in the healthy control-groups. The involvement of intron 3 polymorphism and risk of breast cancer is controversial (Wang-Gohrke et al., 1998; Costa et al., 2007). These results demonstrated that women with A2A2 genotype have an increased risk for developing breast cancer. However, other studies have expressed no correlations between the intron 3 genotypes and the risk of breast cancer (Oliveira., 2005; Costa et al., 2007; Bellini et al., 2012). In this research no association between the intron $3(+16 \mathrm{bp})$ polymorphism and the risk of developing breast cancer were noted. In TP53 PIN3 polymorphism, the findings of this study demonstrated no correlation of A2A2 genotype with sporadic breast cancer. Moreover, in this study, the frequency of A1allele was $72 \%$ and A2 was $28 \%$ in the breast cancer patients and frequency of 
these allele in the healthy control-groups were $78.5 \%$ and $21.5 \%$ no significant differences were noted, in the breast cancer patients versus the healthy women. We analyzed the combined R72P PIN3 Ins 16 bp polymorphisms in TP53 gene and their association with an increased risk of breast cancer. This investigation did not find any association between these Single-nucleotide polymorphism (SNPs) and breast cancer risk in the affected patients versus the control-groups.

In summation, based on the research and data represented by this study, for TP53 Arg72Pro and $16 \mathrm{bp}$ duplication polymorphisms risk of breast cancer the results demonstrated that the intron3 (+16 bp) and exon 4 (Arg) pairwise haplotypes were not associated with a higher risk of breast cancer in northwest female population. however, this initial project limited as the group sizes were small in number.

\section{Acknowledgements}

Authors would like to thank hematology and oncology research center,Tabriz university of medical science, Tabriz, Iran for supporting this (project No: 92/21) which was a part of MSc thesis No: 92/2-7/4.

\section{References}

Ainsley W and James H (1997). Polymorphisms of H-ras-1 and p53 in breast cancer and lung cancer:a meta-analysis. Environ Health Perspect, 4, 919-26.

Alawadi S, Ghabreau L, Alsaleh M, Abdulaziz Z, et al (2011). P53 gene polymorphisms and breast cancer risk in Arab women. Med Oncol, 28, 709-15.

Bellini MF, Cadamuro AC, Succi M, Proenca MA, et al (2012). Alterations of the TP53 gene in gastric and esophageal carcinogenesis. J Biomedicine Biotechnol, 2012, 89196.

Bergamaschi D, GascoM,Hiller L, et al(2003).p53 polymorphism influences response in cancer chemotherapy via modulation of p73-dependent apoptosis. Cancer Cell, 3, 387-402.

Berrada N, Amzazi S, Ameziane-El-Hassani R, et al (2013). No evidence of correlation between $\mathrm{p} 53$ codon 72 polymorphism and risk of bladder cancer in Moroccan patients. Clin Cancer Invest J, 1, 302-6.

Costa S, Pinto D, Pereira D, et al (2008). Importance of TP53 codon 72 and intron 3 duplication 16bp polymorphisms in prediction of susceptibility on breast cancer. BMC cancer. $\mathbf{8}, 32$.

Guleria K, Sharma S, Manjari M, et al (2012). p.R72P, PIN3 Ins 16bp polymorphisms of tp53 and ccr5?32 in north indian breast cancer patients. Asian Pac J Cancer Prev, 13, 3305-11.

Hrstka R, Beranek M, Klocova K, et al(2009).Intronic polymorphisms in TP53 indicate lymph node metastasis in breast cancer. Oncol Reports, 22, 1205-11

Hu Z, Li X, Yuan R, et al (2010). Three common TP53 polymorphisms in susceptibility to breast cancer, evidence from meta-analysis. Breast Cancer Res Treat, 120, 705-14.

Oliveira A, Ross J, Fletcher J (2005). Tumor suppressor genes in breast cancer, Am J Clin Pathol, 124, 16-28.

Papadakis EN, Dokianakis DN, Spandidos DA (2000). p53 codon 72 polymorphism as a risk factor in the development of breast cancer. Mol Cell Biol Res Communicat, 3, 389-92.

Samson M, Swaminathan R, Rama R, et al (2007). Role of GSTM1 (null/present), GSTP1 (Ile105Val) and P53 (Arg72Pro) genetic polymorphisms and the risk of breast cancer - a case control study from South India. Asian Pac J Cancer Prev, 8, 253-7.

Sharma S, Sambyal V, Guleria K, et al (2014). TP53 polymorphisms in sporadic north indian breast cancer patients. Asian Pac J Cancer Prev, 15, 6871-9.

Siddique M, Balram C, Fiszer-Maliszewska L, et al (2005). Evidence for selective expression of the p53 codon 72 polymorphs: implications in cancer development. Cancer Epidemiol Biomarkers Prev, 14, 2245-52.

Sjalander A, Birgander R, Hallmans G, et al (1996). p53 polymorphisms and haplotypes in breast cancer. Carcinogenesis, 17, 1313-6.

Vijayaraman K, Veluchamy M, Murugesan P, et al (2012). p53 exon 4 (codon 72) polymorphism and exon 7 (codon 249) mutation in breast cancer patients in southern region (Madurai) of Tamil Nadu. Asian Pac J Cancer Prev, 13, 511-6.

Wang-Gohrke S, Rebbeck TR, Besenfelder W, et al (1998). p53 germline polymorphisms are associated with an increased risk for breast cancer in German women. Anticancer Res, 18, 2095-9.

Whibley C, Pharoah PD, Hollstein M (2009). p53 polymorphisms: cancer Implications. Nat Rev Cancer, 9,95-10.

Zhou X, Gu Y, Zhang S (2012). Association between p53 codon 72 polymorphism and cervical cancer risk among Asians: a huge review and meta-analysis. Asian Pac J Cancer Prev, 13, 4909-14. 